\title{
Development of Novel Self-Healing Polymers with High Temperature Stability
}

\author{
Yunseon $\mathrm{Heo}^{1}$ \\ University of Florida, Gainesville, FL 32610 \\ Henry A. Sodano ${ }^{2}$ \\ University of Michigan, Ann Arbor, MI 48109
}

\begin{abstract}
For thermo-responsive self-healing polymers with an intrinsic system, the Diels-Alder (DA)
reaction is one of the most promising mechanisms since it is highly efficient, simple, and is repeatable with only the application of heat. Two new approaches with regards to the DA reaction are developed to improve current self-healing DA polymers. First, by using maleimide and furan moieties as the DA healing agents, new thermo-responsive self-healing polymers are developed that use the force from the shape memory effect to close two crack surfaces instead of external forces. Secondly, by introducing maleimide and anthracene DA moieties, self-healing polymers with increased cleaving reaction temperatures above $250{ }^{\circ} \mathrm{C}$ are developed. This work represents the first demonstration of a polymer network acquiring large and repeatable healing behavior only through the crosslinking DA reaction.
\end{abstract}

\section{Introduction}

$\mathrm{T}$ here has been an increased focus on developing self-healing polymers and composites since all materials have the potential to become damaged or degraded over time by various mechanical, chemical, thermal and UV radiation factors ${ }^{1,2}$. The self-healing ability is especially appealing for polymers and composite materials since these types of materials are often used in large-scale applications and it would be beneficial to be able to repair damaged areas in situ. For example, if an aircraft part made of fiber reinforced polymer (FRP) composites were to become damaged, a healing mechanism such as heat could potentially be employed to heal the damage without removing the part, thus allowing the aircraft to resume operations much faster. Self-healing materials could also be useful in space where the environment adversely affects repair operations. Moreover, self-healing polymers could potentially be used in everyday applications, such as a coating material to protect electronic products (e.g., cell phones and laptops) and automobile parts from minor damages. By incorporating self-healing materials, the life and cost efficiency of products and components can increase ${ }^{3}$.

There are three main different types of healing mechanisms: (1) capsule based, (2) vascular, and (3) intrinsic. Generally, capsule and vascular based healing systems are considered extrinsic systems since they store healing agents in different media that has been embedded into the polymer matrix ${ }^{2,-14}$. Meanwhile, intrinsic systems contain the healing functionality in their matrix and can be healed multiple times. The advantage of an extrinsic system is that healing can be triggered once cracks are induced, but unlike an intrinsic system, the mechanical properties of the healed polymers could be different after integration of the healing agents ${ }^{15}$. Also, for bulk application, the multiple reagents in the separate media could be expensive and difficult to fabricate ${ }^{16}$. However, the intrinsic system can partially or fully repair the original properties of a polymer multiple times without any other remaining additional defects after the healing process. This system can be less complex than the extrinsic systems since variables such as compatibility between healing agents and capsules/tubes, and integration of them, can be neglected in this system ${ }^{3}$. This has resulted in much research being conducted on this system in recent years. Therefore, the intrinsic healing system was chosen in this research and the Diels-Alder (DA) reaction was adapted for the healing mechanism. The DA reaction is a thermally reversible reaction between a conjugated diene and a dienophile, from which the resultant product is a cyclohexene derivative ${ }^{17}$. The DA adducts after crosslinking, but can still undergo a reverse reaction at higher temperatures, which is known as a retro Diels-Alder (rDA) reaction ${ }^{18}$.

${ }^{1}$ Graduate student, Materials Science and Engineering, 1320 Beal Avenue Ann Arbor, MI 48109

${ }^{2}$ Associate professor, Aerospace Engineering and Materials Science and Engineering, 1320 Beal Avenue, 3016 FXB, Ann Arbor, MI 48109, and professional AIAA member 
Among all of the dienes and dienophiles, the furan and maleimide groups have been the most commonly selected for use in the synthesis of self-healing polymers ${ }^{19-22}$. Also, the anthracene and maleimide groups have recently been studied to synthesize higher thermal stability self-healing polymers. This is because the only limitation of the diene used in the DA reaction is for it to be s-cis conformation (the double bonds are cis about the single bond, s= single ${ }^{23}$; however, the cis conformation is thermodynamically less stable due to steric repulsion, especially when the substituents are larger than hydrogen. Thus, the furan group is a very reactive diene since the cis conformation is already locked in its structure. Similar to the furan group, the anthracene group is an appropriate candidate as a diene due to the fixed cis conformation. For a dienophile monomer, maleimide groups are typically chosen. In order to increase the reactivity of the dienophile, it is necessary to have one or more electron-withdrawing groups, such as a carbonyl group $(\mathrm{C}=\mathrm{O})$ or a cyano group $(\mathrm{C} \equiv \mathrm{N})$, attached to the $\mathrm{C}=\mathrm{C}$ bond. The role of electron-withdrawing groups are to withdraw electrons from the $\mathrm{C}=\mathrm{C}$ bond and to make the $\mathrm{sp} 2$ carbon have a partially positive charge. This partially positive charge helps the dienophile be more reactive toward the diene groups ${ }^{23}$. Therefore, maleimide moieties with two $\mathrm{C}=\mathrm{O}$ bonds can be a good dienophile candidate. In theory, the cleaving temperature for the furan and maleimide moieties is approximately $120^{\circ} \mathrm{C}$ while the cleaving temperature between anthracene and maleimide is above $250{ }^{\circ} \mathrm{C}^{24-26}$.

In this work, two different diene molecules were employed, along with same dienophile, to demonstrate the selfhealing ability. For the first group of self-healing polymers, three different molar ratios of the DA molecules of furfuryl alcohol and (hydroxylethyl)-maleimide were used to synthesis polyurethanes. In these polyurethanes, the shape memory effect was utilized to close the crack surfaces instead of external pressures. For the second group of self-healing polymers, the monomers of 9-anthracenemethanol and (hydroxylethyl)-maleimide were used to synthesize two different novel polyurethanes with higher thermal stability.

\section{Experimental Methods}

\section{A. Materials}

Commercial furfuryl alcohol (FA), 9-anthracenemethanol, triethanolamine (TEA), N,N,N',N'tetrakis(hydroxypropyl)ethylenediamine (HPED), hexamethylene diisocyanate (HDI) and all chemicals for the $\mathrm{N}$-(2Hydroxyethyl)-maleimide (HEM) synthesis (maleic anhydride, furan, ethanolamine, methanol, and toluene) were ACS certified grade and used as received.

\section{B. Monomer Synthesis}

The HEM monomer (3) was synthesized through a three step process as shown in Figure 1 and this was necessary to protect the $\mathrm{C}=\mathrm{C}$ bonds in maleic anhydride with furan since the $\mathrm{C}=\mathrm{C}$ bonds can react with the amine functional group from ethanolamine. The first reaction was carried out in toluene at room temperature by using the DA reaction and the resulting compound (1) was precipitated out at room temperature once the reaction had reached completion and been vacuum filtered. Compound (1) was then reacted with ethanolamine in an ice bath for 30 minutes, followed by refluxing in methanol for 24 hours. Upon completion of the reaction, the mixture was cooled down to room temperature and the resulting

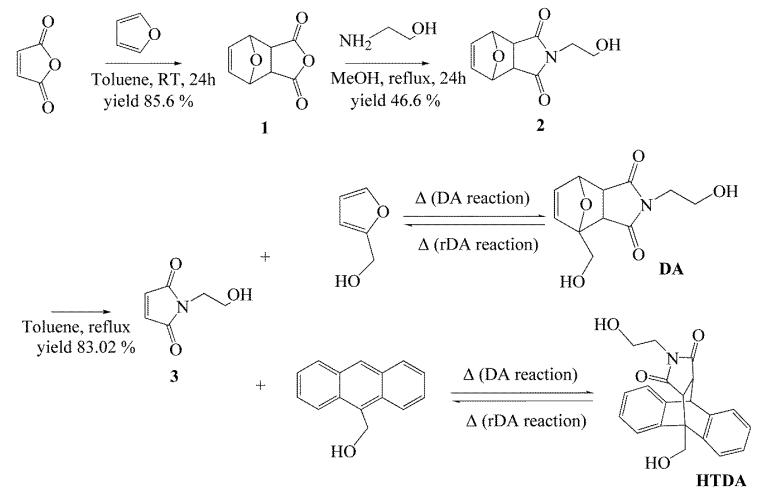

Figure 1. A schematic of the healable monomers' (DA and HTDA) syntheses procedures.

compound (2) was crystallized and vacuum filtered without any further purification process. Then, the furan was removed through the $\mathrm{rDA}$ reaction by refluxing the solution in toluene until the peaks for the furan molecule were no longer detected at $6.53 \mathrm{ppm}$ and $5.10 \mathrm{ppm}$ with proton nuclear magnetic resonance ( ${ }^{1} \mathrm{H}$ NMR). Finally, the solution was cooled down and the HEM was crystallized from the toluene.

In order to confirm that all DA molecules were crosslinked before testing was performed, for the first group of the self-healing polymers, the HEM and FA were precrosslinked at $75^{\circ} \mathrm{C}$ for 12 hours in toluene. Once the HEM and FA were completely crosslinked, the DA monomer was precipitated out from the toluene solvent at $75^{\circ} \mathrm{C}$, thus the resulting compound could be vacuum filtrated and washed with diethyl ether. Also, HTDA was prepared by the precrosslinking reaction between the HEM and 9-anthracenemethanol at $110{ }^{\circ} \mathrm{C}$ for 24 hours in toluene. After 24 hours, the hot solution was vacuum filtered to remove possible impurities and then, the HTDA monomer was precipitated out once the solution was cooled down to room temperature. After, the precipitation (HTDA) was filtered and dried in a vacuum oven. 


\section{Polymer Synthesis}

A total of five self-healing polyurethanes were synthesized by using the two different healable monomers (DA and HTDA). The first three polyurethanes (1DA1T, 1.5DA1T, 2DA1H) were synthesized with DA, TEA/HPED, and HDI monomers. For 1DA1T, 1 mole of the DA monomer, 1 mole of TEA, and 2.5 moles of HDI were used. For 1.5DA1T, 1.5 moles of the DA monomer, 1 mole of TEA, and 3 moles of HDI were used. Similarly, 2 moles of the DA monomer, 1 mole of HPED, and 4 moles of HDI were used when synthesizing 2DA1H. To synthesize all the polymers, the DA

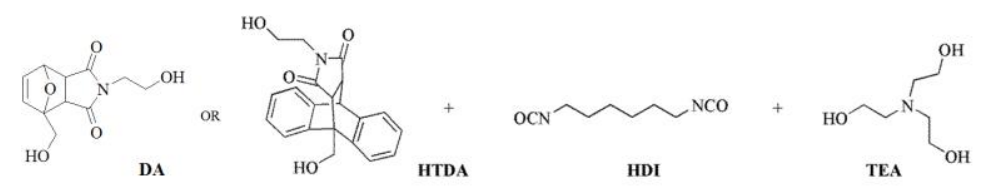
monomer was melted at $105{ }^{\circ} \mathrm{C}$ and then mixed with the rest of monomers (TEA, HPED, and HDI) at the same temperature. Next, the mixture was poured into a Teflon mold and placed into an oven preheated to $100{ }^{\circ} \mathrm{C}$ under nitrogen conditions. The temperature was then increased to $125^{\circ} \mathrm{C}$ over one hour with a two hour dwell time. After, the specimen was deceased to $90{ }^{\circ} \mathrm{C}$ over one hour and kept at this temperature for two hours before finally cooling down to $70{ }^{\circ} \mathrm{C}$ over one hour with a two hour dwell time. The polymerization temperature profile is designed to maximize the amount of crosslinking in the polymer after the reaction has reached completion. This polymerization process with the HEM monomer and the polyurethane structure is shown in Figure 2. The second group self-healing polymers (0.5HTDA1T and 0.8HTDA1T) were also synthesized with HTDA, TEA, and HDI as shown in Figure 2. To produce 0.5 HTDA1T, 0.5 moles of HTDA, 1 mole of TEA, and 2 moles of HDI were reacted. Similarly, to produce 0.8HTDA1T, 0.8 moles of HTDA, 1 mole of TEA, and 2.3 moles of HDI were reacted. During the synthesis of both polymers, the proper molar ratio of HTDA and HDI were reacted at $105{ }^{\circ} \mathrm{C}$ for approximately 3 hours until the mixture became completely transparent. The molten resin was mixed with TEA until it became one phase and then was quickly poured into a Teflon mold. The mold with resin was degassed for 30 minutes in a vacuum oven at room temperature. Then, the specimen was heated up to $125{ }^{\circ} \mathrm{C}$ over one hour and kept at this temperature for two hours. After, the specimen was decreased to $90{ }^{\circ} \mathrm{C}$ over one hour with a two hour dwell time and finally cooled down to $70{ }^{\circ} \mathrm{C}$ over one hour and kept for another two hours.

\section{Crosslinking DA Reaction}

The crosslinking kinetics of the DA reaction for furan/maleimide and anthracene/maleimide were characterized through variable temperature proton nuclear resonance (VT ${ }^{1} \mathrm{H}$ NMR) with a $500 \mathrm{MHz}$ magnetic field at $140{ }^{\circ} \mathrm{C}$. The kinetics are important to evaluate since the crosslinking efficiency of these two molecules can be directly related to the overall healing efficiency because the mechanically cleaved bonds from testing need to be reformed to heal the crack surfaces. For the NMR characterization process, two samples were prepared. The first sample consisted of 1 mole of the HEM and 1 mole of FA and the second sample with 1 mole of HEM and 1 mole of 9anthracenemethanol. Each sample was dissolved in DMSO- $\mathrm{d}_{6}$ solvent in a NMR tube. Then, the mixtures were heated to $140{ }^{\circ} \mathrm{C}$ by using an internal heating function of the NMR instrument to measure the changes in the peaks until the peaks did not show any changes.

\section{E. Thermal Analysis}

The thermal stability of the polymers was investigated through thermogravimetric analysis (TGA, TA Q50) to measure decomposition of the polymers. The TGA testing was conducted at a heating rate of $20{ }^{\circ} \mathrm{C} / \mathrm{min}$ up to 600 ${ }^{\circ} \mathrm{C}$ under a nitrogen condition. Approximately $10 \mathrm{mg}$ of sample was placed on the platinum pan and the balance was purged at $40 \mathrm{~mL} / \mathrm{min}$ and the sample was purged at $60 \mathrm{~mL} / \mathrm{min}$. All the acquired data from TGA was analyzed with "TA Universal Analysis" software offered by TA instruments.

\section{F. Healing Performance}

The healing efficiency of each polyurethane was measured to validate the healing abilities of these developed polymers. The healing efficiency was calculated through compact tension (CT) fracture testing in order to obtain the virgin and post-healed maximum loads after cracks were induced. The healing efficiency is equal to the post-healing maximum load divided by the initial maximum load multiplied by 100. Each polyurethane were prepared following 
a slightly modified ASTM D 5045 standard configuration as shown in Figure 3A. Two modifications were then applied, including the addition of a resting hole along the crack path to terminate the propagation in the middle of the sample and the notch length to enhance the shape memory effect. Termination of the crack via the resting hole was needed since the shape memory effect arises from the portion of the sample that is left intact and the polymers exhibit uncontrolled crack growth in their glassy state.

\section{G. Influence of Shape Memory Effect}

As previously mentioned, each specimen was prepared with two modifications from the ASTM D 5045 standard CT specimen dimensions and one modification was to shorten the pre-crack length to enhance the shape
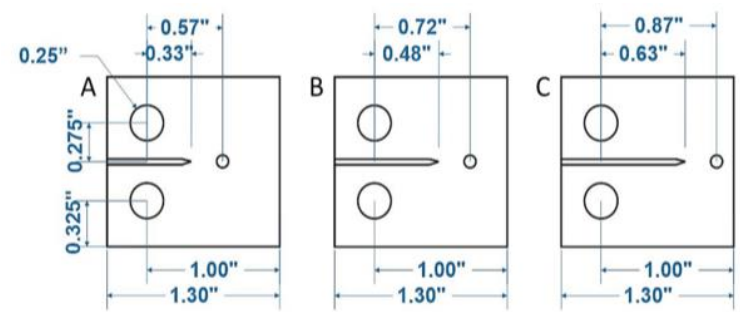

Figure 3. Three different Types (A, B, and C) of CT test specimens with varying pre-crack lengths. memory recovery force since the shape memory effect arises from the unbroken region. Therefore, additional testing was designed and conducted in order to determine the impact that the size of the unbroken area has on the shape memory recovery force, and ultimately the healing efficiency. As shown in Figure 3, three different CT specimen Types (A, B, and C) with varying pre-crack length were developed and tested. The length of the crack path between the tip of the pre-crack and the resting hole $(0.24$ inches) for these different specimen types was the same.

\section{Results and Discussion}

\section{A. Crosslinking Reaction}

The crosslinking abilities of the two different molecules (DA and HTDA) were characterized since the crosslinking confirms the healing ability of the polymers. This is because the healing occurs by the reformation of the mechanically broken C-C bonds. As shown in Figure 4, the VT proton NMR spectrums indicate the kinetics of the crosslinking between the FA and HEM molecules. The two peaks at 6.52 ppm and $5.08 \mathrm{ppm}$ appeared when the HEM and FA monomers connected at $80{ }^{\circ} \mathrm{C}$. Similar to the DA molecule, proton peaks at $8.5 \mathrm{ppm}, 8.1 \mathrm{ppm}$, and $7.5 \mathrm{ppm}$ for anthracene and at $7 \mathrm{ppm}$ for maleimide were used as crosslinking indicators to confirm the crosslinking ability (as shown in Figure 5). Therefore, the crosslinking efficiency can be easily evaluated between anthracene and maleimide using the 1H NMR spectrum. From Figure 5, it can clearly be seen that the peaks at $8.5 \mathrm{ppm}, 8.1 \mathrm{ppm}$, and $7.5 \mathrm{ppm}$ for the anthracene protons and the peak at $7 \mathrm{ppm}$ for maleimide protons disappeared as the crosslinking reaction occurred. The peak at $7 \mathrm{ppm}$ shifted to $6.9 \mathrm{ppm}$ following cycloaddition due to changes in the neighboring protons.

For both molecules, the efficiency for the crosslinking DA reaction between these molecules can be concluded as close to $100 \%$ since the anthracene and maleimide peaks in Figures 4 and 5 essentially disappear from the VT 1H NMR spectrum. However, some peaks still slightly appear in the spectra, indicating that the crosslinking efficiency of the solid state specimens are lower than $100 \%$ because the NMR spectrum were obtained from a liquid state specimen with better molecule mobility. This might help to explain why slight drops in healing efficiency with each repeatable cycle were observed.

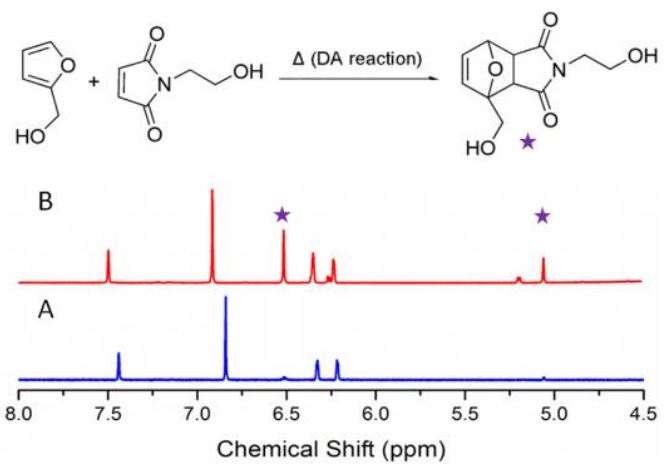

Figure 4. VT ${ }^{1} \mathrm{H}$ NMR spectra for the kinetics of the crosslinking $\mathrm{DA}$ reaction between $\mathrm{FA}$ and HEM, A) before crosslinking and $B$ ) after crosslinking.

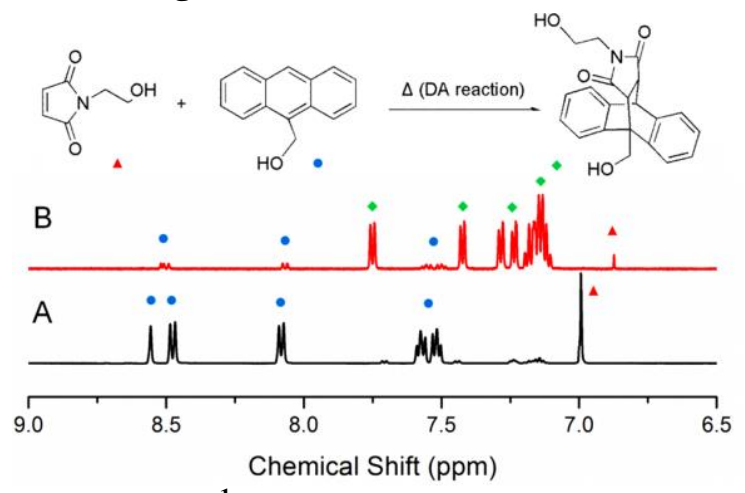

Figure 5. VT ${ }^{1} \mathrm{H}$ NMR spectra for the kinetics of the crosslinking DA reaction between 9anthracenemethanol and HEM, A) before crosslinking and B) after crosslinking. 


\section{B. Thermal Analysis}

All five polymers were tested to measure the decomposition temperatures in order to validate the thermal stability of the polymers. As a result, the TGA curves shown in Figure 6 demonstrate that all the polymers do not decompose until the temperatures are greater than $250{ }^{\circ} \mathrm{C}$. Although beyond the $\mathrm{T}_{\mathrm{g}}$, both polymers are stable for applications up to $250{ }^{\circ} \mathrm{C}$.

\section{Healing Performance}

The healing performances of all the polymers were verified through compact tension (CT) testing. From the CT testing, the maximum loads at fracture were obtained and used to calculate the efficiency as the maximum loads of the post-healing specimens divided by the maximum loads of the initial specimens.

Following fracture of the specimen, the healing was performed in different ways for two different groups of self-healing polymers. For the first group with FA and HEM DA molecules (1DA1T, 1.5DA1T, and 2DA1H), after a crack was formed in a specimen, and without the use of an external force to close the crack, the CT specimen was placed face-down in a vacuum oven under a nitrogen environment such that no gravitational forces contributed to the crack closure. The oven was slowly heated up to $135{ }^{\circ} \mathrm{C}$ over 2.5 hours and kept at this temperature for two hours. Then, the specimen was cooled down to $90{ }^{\circ} \mathrm{C}$ over one hour and kept at this temperature for two hours and finally cooled to $70{ }^{\circ} \mathrm{C}$ over one hour and kept again at this temperature for another two hours. For the second group, the healing was performed in a hot press with approximately $100 \mathrm{psi}$ of pressure applied. The specimen was heated for two hours at $160{ }^{\circ} \mathrm{C}, 120{ }^{\circ} \mathrm{C}$, and lastly $70{ }^{\circ} \mathrm{C}$. The specimen was then removed from the press and heated at $120{ }^{\circ} \mathrm{C}$ in an oven under ambient pressure to recover the shape and to eliminate any internal stresses that might have been created from the pressure.

The average healing efficiencies of all five polymers are provided in Table 1. For the first and the second healing cycles, efficiencies of $79.8 \%$ and $69.3 \%$ for 1DA1T, $84.1 \%$ and $84.3 \%$ for $1.5 \mathrm{DA} 1 \mathrm{~T}$, and $96.1 \%$ and $82.1 \%$ for 2DA1H, $66.3 \%$ and $68.2 \%$ for $0.5 \mathrm{HTDA} 1 \mathrm{~T}$, and $94.4 \%$ and $77.8 \%$ for $0.8 \mathrm{HTDA} 1 \mathrm{~T}$ were obtained, respectively. Based on these results, the polymer that exhibited the higher and more stable healing performance is $2 \mathrm{DA} 1 \mathrm{H}$. This result is attributed to the greater amount of available furan and maleimide molecules to heal the crack surfaces, when compared to 1DA1T and 1.5DA1T. Also, the DA molecules are smaller than the HTDA molecules and therefore, the steric effect in the polymer stucture is smaller.

\section{Influence of Shape Memory Effect}

Three different types of 1.5DA1T specimen were tested to obtain the initial and post-healed maximum loads at fracture, and these measured loads were used to calculate the healing efficiencies for three healing cycles. As shown in Table 2, the average healing efficiencies for the Type $\mathrm{C}$ specimen were the lowest and when observed, the healed area on the specimen only came close to the resting hole after the healing process. This indicates that the shape memory recovery force was not sufficient to completely close the crack surfaces, resulting in the broken DA bonds not reforming. When comparing the efficiencies between the Type A and Type B samples, the first healing cycle

5

American Institute of Aeronautics and Astronautics
Table 2. The average healing efficiencies and standard deviations (SD) for three different 1.5DA1T specimen Types (A, $\mathrm{B}$, and C).

\begin{tabular}{ccccccc}
\hline & \multicolumn{2}{c}{ 1.5DA1T-A } & \multicolumn{2}{c}{ 1.5DA1T-B } & \multicolumn{2}{c}{ 1.5DA1T-C } \\
\hline $\begin{array}{c}\text { Healing } \\
\text { Cycle }\end{array}$ & $\begin{array}{c}\text { Healing } \\
\text { Efficiency }\end{array}$ & SD & $\begin{array}{c}\text { Healing } \\
\text { Efficiency }\end{array}$ & SD & $\begin{array}{c}\text { Healing } \\
\text { Efficiency }\end{array}$ & SD \\
\hline $1^{\text {st }}$ & $84.1 \%$ & 14.5 & $83.7 \%$ & 5.7 & $67.8 \%$ & 11.1 \\
$2^{\text {nd }}$ & $84.3 \%$ & 7.8 & $61.7 \%$ & 14.5 & $48.4 \%$ & 19.6 \\
\hline
\end{tabular}


efficiencies are similar to each other but for the second healing cycle, the Type B specimen had a much larger decrease. This indicates that the Type B specimen was unable to provide a satisfactory amount of shape memory recovery force needed for repeatable healing performance.

\section{Conclusions}

The first part of this work demonstrated that polymers combining two functionalities (self-healing and shape memory) can be developed. These functionalities eliminate the need to apply an external force to close a crack during the healing process. The kinetics of the crosslinking between furan and maleimide were verified through proton VT NMR analysis. Two proton peaks at $5.08 \mathrm{ppm}$ and $6.52 \mathrm{ppm}$ were observed, indicating that the protons of the newly formed $\sigma \mathrm{C}-\mathrm{C}$ bonds appeared after crosslinking at $80^{\circ} \mathrm{C}$. Finally, the healing efficiencies calculated using maximum fracture loads from CT testing confirmed the repeatable healing. The healing efficiencies of $80 \%$, $84 \%$, and $96 \%$ were calculated for the first healing cycle for 1DA1T, 1.5DA1T and 2DA1H, respectively, and only a slight loss in healing efficiencies were seen with each subsequent cycle. The thermally reversible form of the DA reaction placed a limitation on the temperature range for which these polymers can be used. Hence, the DA reaction between anthracene and maleimide moieties was investigated for the healing mechanism. In order to confirm a thermal stability of up to $250{ }^{\circ} \mathrm{C}$, TGA was conducted. The TGA data indicated that the polymers were stable up to approximately $250{ }^{\circ} \mathrm{C}$ for all five polymers. These data are significant since it has been shown that these polymers can be used in applications that require thermal stability up to $240^{\circ} \mathrm{C}$. Moreover, the efficiencies of the crosslinking reactions were analyzed in solvent with VT ${ }^{1} \mathrm{H}$ NMR and the reactions proceeded to roughly $100 \%$ completion at $140{ }^{\circ} \mathrm{C}$. Then, the healing efficiencies were calculated using CT fracture testing and values of $66 \%$ for $0.5 \mathrm{HTDA} 1 \mathrm{~T}$ and $94 \%$ for 0.8 HTDA1T were obtained for the first healing cycle. However, these polymers needed to be compressed by external forces during the healing process in order to obtain the healing performance, unlike the initially investigated polymers (1DA1T, 1.5DA1T, and 2DA1H).

\section{Acknowledgment}

The authors gratefully acknowledge the support from the Army Research Office (Award \#: W911NF-12-1-0014) and the National Science Foundation (Grant \# CMMI- 1132416).

\section{References}

${ }^{1}$ Osswald, T. and Menges, G. Materials science of polymers for engineers. $2^{\text {nd }}$ ed., Hanser Gardner Publications, Cincinnati, 2003.

${ }^{2}$ Wu, D. Y., Meure, S., and Solomon, D. "Self-healing polymeric materials: A review of recent developments," Progress in Polymer Science, 33, 479-522, 2008.

${ }^{3}$ Blaiszik, B., Kramer, S., Olugebefola, S., Moore, J. S., Sottos, N. R., and White, S. R. "Self-healing polymers and composites," Annual Review of Materials Research, 40, 179-211, 2010.

${ }^{4}$ White, S. R. et al. "Autonomic healing of polymer composites," Nature, 409, 794-797, 2001.

${ }^{5}$ Jones, A. S., Rule, J. D., Moore, J. S., White, S. R., and Sottos, N. R. "Catalyst morphology and dissolution kinetics of selfhealing polymers," Chemistry of Materials, 18, 1312-1317, 2006.

${ }^{6}$ Liu, X., Lee, J. K., Yoon, S. H., and Kessler, M. R. "Characterization of diene monomers as healing agents for autonomic damage repair," Journal of applied polymer science, 101, 1266-1272, 2006.

${ }^{7}$ Cho, S. H., Andersson, H. M., White, S. R., Sottos, N. R., and Braun, P. V. "Polydimethylsiloxane-Based Self-Healing Materials," Advanced Materials, 18, 997-1000, 2006.

${ }^{8}$ Carolyn, D. "Matrix cracking repair and filling using active and passive modes for smart timed release of chemicals from fibers into cement matrices," Smart Materials and Structures, 3, 118, 1994.

${ }^{9}$ Bergman, S. D., and Wudl, F. "Mendable polymers," Journal of Materials Chemistry, 18, 41-62, 2008.

${ }^{10}$ Wool, R. P. "Self-healing materials: a review," Soft Matter, 4, 400-418, 2008.

${ }^{11}$ Bleay, S., Loader, C., Hawyes, V., Humberstone, L., and Curtis, P. “A smart repair system for polymer matrix composites," Composites Part A: Applied Science and Manufacturing, 32, 1767-1776, 2001.

${ }^{12}$ Toohey, K. S., Sottos, N. R., Lewis, J. A., Moore, J. S., and White, S. R. "Self-healing materials with microvascular networks," Nature materials 6, 581-585, 2007.

${ }^{13}$ Hansen, C. J., Wu, W., Toohey, K. S., Sottos, N. R., White, S. R., and Lewis, J. A., "Self-Healing Materials with Interpenetrating Microvascular Networks," Advanced Materials 21, 4143-4147, 2009.

${ }^{14}$ Therriault, D., Shepherd, R. F., White, S. R., and Lewis, J. A. "Fugitive Inks for Direct-Write Assembly of Three-Dimensional Microvascular Networks," Advanced Materials, 17, 395-399, 2005. 
${ }^{15}$ White, S. R., Blaiszik, B. J., Kramer, S. L. B., Olugebefola, S. C., Moore, J. S., and Sottos, N. R., "Self-healing Polymers and Composites," American Scientist, 99, 392-399, 2011.

${ }^{16}$ Meure, S., Varley, R. J., Wu, D. Y., Mayo, S., and Nairn, K.; Furman, S., "Confirmation of the healing mechanism in a mendable EMAA-epoxy resin," European Polymer Journal, 48, 524-531, 2012.

${ }^{17}$ Diels, O., and Alder, K. "Syntheses in the hydroaromatic series. I. Addition of diene hydrocarbons," Ann 460, 98-122, 1928.

${ }^{18}$ Rickborn, B. Organic Reactions, John Wiley \& Sons, Inc., New York, 1998.

${ }^{19}$ Fringuelli, F., and Taticchi, A. Dienes in the Diels-Alder reaction, Wiley, New York, 1990.

${ }^{20}$ Kappe, C. O., Murphree, S. S., and Padwa, A. "Synthetic applications of furan Diels-Alder chemistry," Tetrahedron, 53, 14179-14233, 1997.

${ }^{21}$ Adeva, M., Sahagún, H., Caballero, E., Peláez-Lamamié de Clairac, R., Medarde, M., and Tomé, F.,. "Open Analogues of Arcyriaflavin A. Synthesis through Diels-Alder Reaction between Maleimides and 1-Aryl-3-tert-butyldimethylsiloxy-1,3butadienes," The Journal of Organic Chemistry, 65, 3387-3394, 2000.

${ }^{22}$ Barrett, A. G. M., Boffey, R. J., Frederiksen, M. U., Newton, C. G., and Roberts, R. S. "Pyrrolidine synthesis on polystyrene supports: development of a 'one-pot' dipolar cycloaddition strategy," Tetrahedron Letters 42, 5579-5581, 2001.

${ }^{23}$ Bruice, Y. P. Organic Chemistry, Pearson Prentice Hall, New York, 2007.

${ }^{24}$ Liu, Y.-L., and Chuo, T.-W. "Self-healing polymers based on thermally reversible Diels-Alder chemistry," Polymer Chemistry, 2013.

${ }^{25}$ Grigoras, M., and Colotin, G. "Copolymerization of a bisanthracene compound with bismaleimides by Diels-Alder cycloaddition," Polymer International, 50, 1375-1378, 2001.

${ }^{26}$ Jones, J. R., Liotta, C. L., Collard, D. M., and Schiraldi, D. A. "Cross-Linking and Modification of Poly(ethylene terephthalate-co-2,6-anthracenedicarboxylate) by Diels-Alder Reactions with Maleimides," Macromolecules 32, 5786-5792, 1999. 